\title{
HIGHER POINT DERIVATIONS ON COMMUTATIVE BANACH ALGEBRAS, III
}

\author{
by H. G. DALES and J. P. McCLURE*
}

(Received 18th September 1979)

\section{Introduction}

Let $A$ be a commutative Banach algebra with identity 1 over the complex field $\boldsymbol{C}$, and let $d_{0}$ be a character on $A$. We recall that a (higher) point derivation of order $q$ on $A$ at $d_{0}$ is a sequence $d_{1}, \ldots, d_{q}$ of linear functionals on $A$ such that the identities

$$
d_{k}(f g)=\sum_{j=0}^{k} d_{i}(f) d_{k-j}(g)
$$

hold for each choice of $f$ and $g$ in $A$ and $k$ in $\{1, \ldots, q\}$. A point derivation of infinite order is an infinite sequence $\left\{d_{k}\right\}$ of linear functionals such that (1.1) holds for all $k$. A point derivation is continuous if each $d_{k}$ is continuous, totally discontinuous if $d_{k}$ is discontinuous for each $k \geqq 1$, and degenerate if $d_{1}=0$.

Some of the terminology given in the previous paragraph was introduced in our earlier paper (3), where we began a study of the continuity properties of point derivations on commutative Banach algebras. In particular, we asked whether, for a given algebra $A$, there is a function $q \mapsto p(q)$ on the set $\boldsymbol{N}$ of natural numbers such that, whenever $d_{1}, \ldots, d_{p(q)}$ is a point derivation on $A$, the "initial segment" $d_{1}, \ldots, d_{q}$ of order $q$ is necessarily continuous. By algebraic methods, we obtained partial results for a number of algebras (see Theorem 2.3 and Examples 2.5 to 2.8 of (3)), and found for the algebra $C^{(n)}$ of $n$-times continuously differentiable functions on a compact interval a complete description of the point derivations, including a determination of the function $p(q)$. In a second paper (4), we continued the study by giving a construction which showed that, in general, the function $p(q)$ need not exist: a consequence of the existence of $p(q)$ is that every point derivation of infinite order is continuous, and we constructed algebras of various types with totally discontinuous point derivations of infinite order.

The aim of the present note is to improve the partial results given in (3) for some particular algebras. In Section 2, we complete the description of the point derivations on the algebra Lip $X$ of bounded Lipschitz functions on a metric space $X$. In Example 2.8 of (3) we showed that a nondegenerate point derivation at a point $t_{0}$ of $X$ has order at most two. In the present article, we show that there are nondegenerate point derivations of order two on $\operatorname{Lip} X$, and that, if $d_{1}, d_{2}$ is such a derivation, then $d_{1}$ is necessarily continuous. These facts follow from a precise characterisation of those point

\footnotetext{
* Assisted by NSERC Grant A8069.
} 
derivations $d_{1}$ which can belong to higher order point derivations. The study of the continuous point derivations on Lipschitz algebras was begun by Sherbert in (8), and further results concerning continuous point derivations on Lip $X$ when $X$ is a compact plane set are given by O'Farrell in (7).

In Section 3, we consider algebras $D^{n}(X)$ of functions $n$-times continuously differentiable on a compact set $X \subset \boldsymbol{C}$. In Example 2.6 of (3), we showed that, with $X$ the closed unit disc, a nondegenerate point derivation on $D^{n}(X)$ at a boundary point of $X$ has order at most $2 n$. It was observed that the result for the closed unit disc would hold for a wider class of sets, the restrictions having to do with the smoothness of the boundary of $X$. In Section 3 of this paper, we show that for such sets $X$, there are analogues for $D^{n}(X)$ of the various technical results proved for $C^{(n)}$ in Section 3 of (3). Thus, the structure of point derivations on $D^{n}(X)$ at a boundary point of $X$ is formally identical with the corresponding structure for $C^{(n)}$.

\section{Lipschitz algebras}

Let $X$ be a metric space with metric $m$, and let Lip $X$ denote the algebra of bounded functions $f$ such that $\|f\|_{m}<\infty$, where $\|f\|_{m}=\sup \{|f(t)-f(s)| / m(t, s): t, s \in X, t \neq s\}$. With pointwise operations and norm $\|f\|=\|f\|_{\infty}+\|f\|_{m}$, Lip $X$ is a regular commutative Banach algebra. Lip $X$ was studied by Sherbert $(\mathbf{8})$, who gave several characterisations of the bounded point derivations of order one at points of $X$. Motivated by one of those characterisations and by the known facts for algebras of continuously differentiable functions, we shall give a description of those point derivations $d_{1}$ of order one at a point $t_{0}$ of $X$ for which there exists a linear functional $d_{2}$ such that $d_{1}, d_{2}$ is a point derivation of order two at $t_{0}$. (Recall that when such a $d_{2}$ exists, we say that $d_{1}$ belongs to a point derivation of order two.) As a consequence, we shall find that any $d_{1}$ which belongs to a higher order point derivation is necessarily continuous. Again using Sherbert's observations, we shall also show that for some metric spaces $X$ (including $[0,1])$, not every continuous point derivation $d_{1}$ belongs to a point derivation of order two.

For $t, s \in X$ with $t \neq s$, write $\psi(t, s)$ for the difference quotient which assigns to a complex-valued function $f$ on $X$ the value $\psi(t, s)(f)=(f(t)-f(s)) / m(t, s)$, and write $\Psi$ for the set of all such difference quotients. Observe that $f \in \operatorname{Lip} X$ if and only if $f$ is bounded and $\{\psi(f): \psi \in \Psi\}$ is bounded. Each $\psi(t, s)$ determines a continuous linear functional on $\operatorname{Lip} X$, with $\|\psi(t, s)\| \leqq 1$, and any weak-* limit point (in the dual of Lip $X$ ) of $\Psi$ is again continuous, with norm at most 1 . Sherbert $(\mathbf{8}$, Theorem 9.3$)$ showed that, if $t_{0} \in X$ and if $\left\{t_{n}\right\}$ and $\left\{s_{n}\right\}$ are sequences in $X$, each converging to $t_{0}$ with respect to the metric $m$, and with $t_{n} \neq s_{n}$ for all $n$, then any weak-* limit point of the sequence $\left\{\psi\left(t_{n}, s_{n}\right)\right\}$ is a bounded point derivation at $t_{0}$. Moreover, the linear span of all such point derivations is weak-* dense in the space of all bounded point derivations of order one on $\operatorname{Lip} X$ at $t_{0}$. Our first result in this section is related to that description of point derivations.

Lemma 2.1. Let $t_{0} \in X$, and let $\left\{\psi\left(t_{\gamma}, s_{\gamma}\right)\right\}$ be a net of difference quotients converging weak-* to a non-zero linear functional $\delta$. Then $\delta$ is a point derivation at $t_{0}$ if and only if each of the nets $\left\{t_{\gamma}\right\}$ and $\left\{s_{\gamma}\right\}$ converges to $t_{0}$ with respect to the metric on $X$. 
Proof. The sufficiency of the condition is proved in (8, Lemma 9.1). To prove the necessity, observe that an argument of Détraz (5, page 81) shows that $\left\{t_{\gamma}\right\}$ and $\left\{s_{\gamma}\right\}$ converge to $t_{0}$ with respect to the weak-* topology on $X$. Since the weak-* and metric topologies agree on $X$ (8, Proposition 2.1), the lemma follows.

Now, let $t_{0}$ be a fixed point of $X$, and let $d_{1}, d_{2}$ be a point derivation of order two on Lip $X$ at $t_{0}$. We are going to show that $d_{1}$ is continuous by showing that $d_{1}$ is a weak-* limit of difference quotients of a special type. It will be convenient to write $\Psi\left(t_{0}\right)$ for the subset of $\Psi$ consisting of difference quotients $\psi\left(t, t_{0}\right)$, with $t \in X$ and $t \neq t_{0}$.

Theorem 2.2. Let $d_{1}$ be a point derivation of order one on Lip $X$ at $t_{0}$, and suppose $d_{1} \neq 0$. Then $d_{1}$ belongs to a point derivation of order two if and only if some non-zero scalar multiple of $d_{1}$ is a weak-* limit point of $\Psi\left(t_{0}\right)$.

We shall isolate a number of the steps in the proof as lemmas. The methods are based on the known facts for the algebra $C^{(1)}$ of continuously differentiable functions on $[0,1]$. It will be convenient to use the symbol $x$ for the function defined on $X$ by $x(t)=m\left(t, t_{0}\right)$. If the diameter of $X$ is not finite, then we "truncate" $x$ at one, as in $(\mathbf{8}$, page 243 ) to ensure that $x$ is bounded. Then $x \in \operatorname{Lip} X$. For $f \in \operatorname{Lip} X, f / x$ denotes the function defined on $X$ by

$$
(f / x)(t)= \begin{cases}f(t) / x(t) & \left(t \neq t_{0}\right), \\ 0 & \left(t=t_{0}\right) .\end{cases}
$$

If $f\left(t_{0}\right)=0$, then $f / x$ is bounded on $X$. Finally, we write $M$ for the maximal ideal $\left\{f \in \operatorname{Lip} X: f\left(t_{0}\right)=0\right\}$.

Lemma 2.3. $M^{2}=x M$.

Proof. Since $x \in M$, we have $M^{2} \supset x M$. To establish the reverse inclusion, suppose that $f \in M$ and $g \in M$. Then it is easy to see that $f g / x$ is bounded and continuous on $X$, and we have to show that $f g / x$ is Lipschitz. If $t \neq t_{0}$, then $\left|\psi\left(t, t_{0}\right)(f g / x)\right| \leqq$ $\left|f(t) g(t) / x(t)^{2}\right| \leqq\|f\|\|g\|$. Also, if $t \neq s, t \neq t_{0}$, and $s \neq t_{0}$, then

$$
\begin{aligned}
|\psi(t, s)(f g / x)|= & \frac{|x(s) f(t) g(t)-x(t) f(s) g(s)|}{x(s) x(t) m(t, s)} \\
\leqq & \frac{|g(t)||| f(t)-f(s) \mid}{x(t)} \frac{\mid f(t, s)}{m} \\
& +\frac{|f(s)|}{x(s)} \frac{|g(t)|}{x(t)} \frac{|x(s)-x(t)|}{m(t, s)}+\frac{|f(s)|}{x(s)} \frac{|g(t)-g(s)|}{m(t, s)} \\
\leqq & 3\|f\|\|g\| .
\end{aligned}
$$

Thus, $\{\psi(f g / x): \psi \in \Psi\}$ is a bounded set of numbers, and the lemma is proved.

Lemma 2.4. Suppose $f \in \operatorname{Lip} X$ and $f$ vanishes in a metric neighbourhood of $t_{0}$. Then $f \in x^{n} M$ for every positive integer $n$. In particular, $f \in x^{2} M \subseteq M^{3}$. 
Proof. There exists $r>0$ such that $f$ vanishes off $E=\{t: x(t) \geqq r\}$. Let $h(t)=$ dist $(t, E)$. Then $x+h \in \operatorname{Lip} X$ and $x+h$ is invertible, so that $f / x=(x+h)^{-1} f$ belongs to Lip $X$. By induction, $f / x^{n} \in \operatorname{Lip} X_{2}(n \in N)$, and the result follows.

Lemma 2.5. Suppose that $f \in M$, and that $\left\{f(t) / x(t): t \neq t_{0}\right\}$ is bounded away from 0 . Then there exists $g$ in $M$ such that $x^{2}=f g$.

Proof. Define $g=x^{2} / f$ on $X$ by $g(t)=x(t)^{2} / f(t)$ if $t \neq t_{0}$, and $g\left(t_{0}\right)=0$. The hypotheses imply that $g$ is bounded and continuous. The proof that $g$ is Lipschitz is similar to the argument in Lemma 2.3. For example, if $t \neq s, t \neq t_{0}$, and $s \neq t_{0}$, then

$$
\begin{aligned}
\psi(t, s)(g) & =\frac{x(t)^{2} f(s)-x(s)^{2} f(t)}{f(s) f(t) m(t, s)} \\
& =\frac{x(s)}{f(s)} \frac{x(t)}{f(t)} \frac{x(t)^{2} f(s)-x(s)^{2} f(t)}{x(s) x(t) m(t, s)},
\end{aligned}
$$

and the required boundedness follows from the hypotheses and estimates similar to those in the proof of Lemma 2.3.

For each of the following three lemmas, we suppose that $d_{1}, d_{2}$ is a point derivation at $t_{0}$ with $d_{1} \neq 0$.

Lemma 2.6. $d_{1}(x) \neq 0$.

Proof. By assumption, there is $f$ in $M$ with $d_{1}(f) \neq 0$. By Lemma 2.3, $f^{2} / x$ belongs to M. Then, $0 \neq d_{1}(f)^{2}=d_{2}\left(f^{2}\right)=d_{1}(x) d_{1}\left(f^{2} / x\right)$.

Because of Lemma 2.6, we can suppose that $d_{1}(x)=1$. Also, whenever $f$ satisfies the hypotheses of Lemma 2.5 , we can conclude that $d_{1}(f) \neq 0$.

Lemma 2.7. If $f \in \operatorname{ker}\left(d_{1}\right) \cap M$, then $|f| \in \operatorname{ker}\left(d_{1}\right)$.

Proof. $\quad d_{1}(|f|)^{2}=d_{2}\left(|f|^{2}\right)=d_{1}(f) d_{1}(\bar{f})=0$.

Lemma 2.8. Suppose $f \in \operatorname{ker}\left(d_{1}\right) \cap M$. Then there is a non-zero point derivation $\delta$ at $t_{0}$ such that $\delta$ is a weak-* limit point of $\Psi\left(t_{0}\right)$ and $f \in \operatorname{ker}(\delta)$.

Proof. Suppose there is no point derivation satisfying the assertions of the lemma. Then Lemma 2.1 implies that there is no sequence $\left\{t_{n}\right\}$ converging to $t_{0}$ in $X$, and such that $\psi\left(t_{n}, t_{0}\right)(f)=f\left(t_{n}\right) / x\left(t_{n}\right) \rightarrow 0$ as $n \rightarrow \infty$. Thus, there are a neighbourhood $U$ of $t_{0}$ in $X$ and a number $r>0$ such that $|f(t)| / x(t) \geqq r$ for each $t \in U \backslash\left\{t_{0}\right\}$. Let $y(t)=$ $\min \{m(t, U), 1\}$ for each $t$ in $X$, and let $g=|f|+y$. Then $y \in \operatorname{Lip} X$, and $y$ vanishes on $U$, so that $d_{1}(y)=0$, by Lemma 2.4. Therefore $d_{1}(g)=d_{1}(|f|)=0$, by Lemma 2.7 and the assumption that $f \in \operatorname{ker}\left(d_{1}\right) \cap M$. Also, it is clear that $g(t) \neq 0$ if $t \neq t_{0}$. Finally, we claim that $\left\{g(t) / x(t): t \neq t_{0}\right\}$ is bounded away from zero. To see this, let $U_{1}=$ 
$\{t \in X:|f(t)| / x(t)<r / 2\}$, and let $r_{1}=m\left(U, U_{1}\right)$. Then, for $t \neq t_{0}$,

$$
\begin{aligned}
g(t) / x(t) & =(|f(t)|+y(t)) / x(t) \\
& \geqq|f(t)| / x(t)+y(t) /\|x\|_{\infty} \\
& \geqq \min \left(r / 2, r_{1} /\|x\|_{\infty}\right),
\end{aligned}
$$

so that the claim will be established if we can show that $r_{1}>0$. If that is not so, there are sequences $\left\{t_{n}\right\}$ in $U$ and $\left\{s_{n}\right\}$ in $U_{1}$ such that $m\left(t_{n}, s_{n}\right) \rightarrow 0$ as $n \rightarrow \infty$. Then

$$
\begin{aligned}
\frac{\left|f\left(t_{n}\right)\right|-\left|f\left(s_{n}\right)\right|}{m\left(t_{n}, s_{n}\right)} & \geqq \frac{r x\left(t_{n}\right)-(r / 2) x\left(s_{n}\right)}{m\left(t_{n}, s_{n}\right)} \\
& =\frac{r}{2}\left[\frac{x\left(t_{n}\right)}{m\left(t_{n}, s_{n}\right)}+\frac{\left(x\left(t_{n}\right)-x\left(s_{n}\right)\right)}{m\left(t_{n}, s_{n}\right)}\right] .
\end{aligned}
$$

Therefore $x\left(t_{n}\right) / m\left(t_{n}, s_{n}\right) \leqq 2\|f\| / r+\|x\|$. Since $m\left(t_{n}, s_{n}\right) \rightarrow 0$, it follows that $t_{n} \rightarrow t_{0}$, and then that $s_{n} \rightarrow t_{0}$. This contradicts the properties of $U$ and $U_{1}$, and so $r_{1}>0$.

Now we can apply Lemma 2.5 to $g$, and conclude that there is $h$ in $M$ such that $x^{2}=g h$. But then, using Lemma 2.6, $0 \neq d_{1}(x)^{2}=d_{2}\left(x^{2}\right)=d_{1}(g) d_{1}(h)$. Therefore $d_{1}(g) \neq 0$. That is a contradiction, so the lemma is proved.

Proof of Theorem 2.2. First we prove the sufficiency. Thus, suppose that $\delta$ is a non-zero point derivation at $t_{0}$, and that $\delta$ is the weak-* limit of a net $\left\{\psi\left(t_{\gamma}, t_{0}\right)\right\}$ from $\Psi\left(t_{0}\right)$. From Lemma 2.1, we conclude that the net $\left\{t_{\gamma}\right\}$ converges in $X$ to $t_{0}$. It follows from this that $\delta(x)=\lim _{\gamma} x\left(t_{\gamma}\right) / m\left(t_{\gamma}, t_{0}\right)=1$. To prove that $\delta$ belongs to a point derivation of order two, it is sufficient to find a linear functional $\lambda$ on $M$ such that $\lambda(f g)=\delta(f) \delta(g)$ whenever $f$ and $g$ belong to $M$ (see (3)). By Lemma 2.3, $f \mapsto f / x$ defines a mapping from $M^{2}$ to $M$. This mapping is clearly linear, and therefore there is a linear functional $\lambda$ on $M$ such that $\lambda(f)=\delta(f / x)$ whenever $f \in M^{2}$. Given $f$ and $g$ belonging to $M$, we can write $f=\delta(f) x+F$ and $g=\delta(g) x+G$, where $F$ and $G$ belong to $\operatorname{ker}(\delta) \cap M$. So $f g / x=\delta(f) \delta(g) x+\delta(f) G+\delta(g) F+F G / x$, and therefore $\lambda(f g)=$ $\delta(f g / x)=\delta(f) \delta(g)$, provided that $\delta(F G / x)=0$. But, since $\left\{t_{\gamma}\right\}$ converges to $t_{0}$ in $X$, we have $x\left(t_{\gamma}\right)=m\left(t_{\gamma}, t_{0}\right)$ for "large" $\gamma$, and therefore $\delta(F G / x)=\lim _{\gamma} F\left(t_{\gamma}\right) G\left(t_{\gamma}\right) / x\left(t_{\gamma}\right)^{2}=0$, since $0=\delta(F)=\lim _{\gamma} F\left(t_{\gamma}\right) / x\left(t_{\gamma}\right)$, and similarly for $G$.

Now we prove the converse. Let $d_{1}, d_{2}$ be a point derivation of order two at $t_{0}$, with $d_{1} \neq 0$. By Lemma 2.6 , we can suppose that $d_{1}(x)=1$. It will be sufficient to show that each weak-* neighbourhood of $d_{1}$ contains a weak-* limit point of $\Psi\left(t_{0}\right)$. Suppose that finitely many functions $f_{1}, \ldots, f_{n}$ from $\operatorname{Lip} X$ are given. For $i=1, \ldots, n$, we can write $f_{i}=f_{i}\left(t_{0}\right) 1+d_{1}\left(f_{i}\right) x+F_{i}$, where $F_{i} \in \operatorname{ker}\left(d_{1}\right) \cap M$. By Lemma 2.7, $\Sigma_{i}\left|F_{i}\right| \in \operatorname{ker}\left(d_{1}\right) \cap M$. By Lemma 2.8 , there is $\delta$, a non-zero point derivation at $t_{0}$ and a weak-* limit point of $\Psi\left(t_{0}\right)$, such that $\delta\left(\Sigma_{i}\left|F_{i}\right|\right)=0$. It is clear that for such a $\delta, f \in M$ and $f \geqq 0$ imply that $\delta(f) \geqq 0$. Therefore, $\delta\left(\left|F_{i}\right|\right)=0$, and hence $\delta\left(F_{i}\right)=0$ for $i=1, \ldots, n$. From the definition of $\delta$ and the fact that $m\left(t_{\gamma}, t_{0}\right)=x\left(t_{\gamma}\right)$ for large $\gamma$, we have $\delta(1)=0$ and $\delta(x)=1$. Therefore $\delta\left(f_{i}\right)=d_{1}\left(f_{i}\right)$ for $i=1, \ldots, n$. So, $\delta$ belongs to the weak-* neighbourhood of $d_{1}$ determined by $f_{1}, \ldots, f_{n}$ and any positive constant, and that proves the theorem.

Since $\Psi\left(t_{0}\right)$ is norm-bounded in $(\operatorname{Lip} X)^{\prime}$, the following corollary is an immediate consequence of Theorem 2.2. 
Corollary 2.9. If $d_{1}, d_{2}$ is a point derivation of order two on $\operatorname{Lip} X$ at a point $t_{0}$ of $X$, then $d_{1}$ is continuous.

As we remarked earlier, there are metric spaces $X$ and continuous point derivations of order one on Lip $X$ which belong to no point derivation of order two. For $X$ the closed unit interval $[0,1]$, that is implied by our Theorem 2.2 and the remark on page 266 of $(8)$ that the weak-* limit points of $\Psi(0)$ do not generate all the bounded point derivations at 0 . In fact, in the general setting, there will exist continuous point derivations of order one belonging to no point derivation of order two as long as the range of the function $t \mapsto x(t)$ contains an interval $[0, r]$ for some $r>0$, for then examination of the function $t \mapsto x(t)^{2} \sin (1 / x(t))$ shows that there are continuous point derivations at $t_{0}$ which are not (multiples of) weak-* limit points of $\Psi\left(t_{0}\right)$. Recall that the situation is different for the algebra $C^{(1)}$ : by $(3)$, Theorem 3.4 , a point derivation of order one on $C^{(1)}$ belongs to a point derivation of order two if and only if it is continuous.

\section{Differentiable functions on plane sets}

In this section, we discuss an algebra, $D^{n}(X)$, of differentiable functions introduced in (2).

Let $X$ be a perfect, compact plane set. A complex-valued function $f$ on $X$ is differentiable at a point $\zeta_{0}$ of $X$ if

$$
f^{\prime}\left(\zeta_{0}\right)=\lim \left\{\frac{f(\zeta)-f\left(\zeta_{0}\right)}{\zeta-\zeta_{0}}: \zeta \rightarrow \zeta_{0}, \zeta \in X\right\}
$$

exists, and $f$ is differentiable on $X$ if it is differentiable at each point of $X$. If $n \in N$, let $D^{n}(X)$ denote the set of functions with continuous $n$th derivative on $X$.

The set $X$ is regular if it is connected by rectifiable arcs and if the geodesic metric on $X$ is equivalent to the Euclidean metric on $C$. In this case, for each $\zeta_{0}$ in $X$, there exists a constant $C_{\zeta_{0}}$ such that each point $\zeta$ of $X$ can be joined to $\zeta_{0}$ by an arc lying in $X$ of length at most $C_{\varepsilon_{0}}\left|\zeta-\zeta_{0}\right|$. The proof of Theorem 1.6 of (2) shows that, if $X$ is regular, then $D^{n}(X)$ is a Banach algebra with respect to pointwise algebraic operations and the norm

$$
\|f\|=\sum_{k=0}^{n} \frac{1}{k !}\left\|f^{(k)}\right\|_{\infty} \quad\left(f \in D^{n}(X)\right) .
$$

We wish to investigate for more general sets $X$ the questions which were answered in (3) in the special case $X=[0,1]$. Thus, we take $X$ to be a regular plane set. In particular, we have in mind the case when $X=\bar{\Delta}$, the closed unit disc.

Clearly, everything is straightforward at interior points of $X$ : functions of $D^{n}(X)$ are analytic on the interior of $X$, and so it is easy to see that all point derivations are continuous and are, up to certain algebraic transformations, ordinary derivatives at the point in question. Thus, we now assume that we are investigating point derivations at a point, say 0 , of the boundary of $X$. 
Using an analogous notation to that of (3), we set, for $k=0, \ldots, n$,

$$
\delta_{k}(f)=\frac{1}{k !} f^{(k)}(0) \quad\left(f \in D^{n}(X)\right)
$$

and

$$
M_{n, k}=\left\{f \in D^{n}(X): f^{(j)}(0)=0 \quad(j=0, \ldots, k)\right\} .
$$

Also, we write $z$ for the coordinate functional on $X$.

An examination of $\S 3$ of (3) shows that, in addition to general algebraic facts about point derivations, the technicalities required for the proofs can be arranged in four groups:

(i) the use of l'Hôpital's rule and Taylor expansions in Lemma 3.2 and the easy parts of Theorem 3.1 of (3);

(ii) the density of polynomials in the algebra, so that, in Theorems 3.3 and 3.4 , a continuous linear functional is determined by its values on polynomials;

(iii) the ability, in Theorem 3.3, to replace a given function $f$ by another function, say $h$, so that $h$ vanishes only at 0 and so that certain point derivations take the same values at $f$ and $h$;

(iv) the harder part of Theorem 3.1(i), namely, the inclusion $z^{n} M_{n, n} \subset M_{n, n}^{2}$.

We shall now consider hypotheses on the set $X$ which will guarantee that the analogous computations can be carried out in $D^{n}(X)$.

First, we observe that the proof of Lemma 1.5 in (2) shows that, if $X$ is regular, then each $f$ in $D^{n}(X)$ has an $n$-Taylor expansion at 0 , so that $f=\sum_{k=0}^{n} \delta_{k}(f) z^{k}+R_{n} f$, where $R_{n} f(\zeta)=o\left(|\zeta|^{n}\right)$ as $\zeta \rightarrow 0, \zeta \in X$. This, in turn, guarantees that all the necessary analogues of l'Hôpital's rule are valid. Thus, the technicalities of group (i) will still apply because of our assumption that $X$ is regular.

Secondly, to ensure that polynomials are dense in $D^{n}(X)$, we assume that $X$ does not separate the plane. Then, given $f$ in $D^{n}(X)$, Mergelyan's theorem shows that $f^{(n)}$ can be uniformly approximated on $X$ by polynomials, and it follows from Lemma 1.5(i) of (2) that suitably chosen $n$th antiderivatives of the polynomials approximating $f^{(n)}$ will approximate $f$ in $D^{n}(X)$.

A specific problem of the type referred to in (iii) was solved in Example 2.6 of (3). There, we showed that, if $f \in D^{n}(\bar{\Delta})$ and $f^{\prime}(1) \neq 0$, then $f=g h$, where $g, h \in D^{n}(\bar{\Delta})$, $g(1) \neq 0$, and $h(\zeta) \neq 0$ if $\zeta \in \bar{\Delta} \backslash\{1\}$. In the proof, we used Theorem 20 of (6). To be able to invoke Nagel's theorem in our present discussion, we make the following assumption:

(A) $X=\bar{U}$, where $U$ is an open set in $C$ and the boundary of $U$ is a finite union of simple rectifiable arcs, each of which is $(n+1)$-times continuously differentiable with respect to arc length.

It is not difficult to see that, if $X$ satisfies condition (A), then $X$ is regular. Using this assumption, we can prove the following result, which can be used whenever a manipulation of type (iii) is required.

Lemma 3.1. Let $X$ be a compact plane set satisfying (A), and let 0 be a boundary point of $X$. Take $k \in\{0,1, \ldots, n-1\}$ and $f$ in $M_{n, k}$ with $\delta_{k+1}(f) \neq 0$. Then there are $g$ and 
$h$ in $D^{n}(X)$ with $f=g h$, where $g(0) \neq 0, h \in M_{n, k}, h(\zeta) \neq 0$ if $\zeta \in X \backslash\{0\}$, and $\delta_{k+1}(h) \neq 0$. Moreover, if $d$ is any first order point derivation at 0 on $D^{n}(X)$, then $d(h)=0$ if and only if $d(f)=0$.

Proof. Since $f \in M_{n, k}$ and $X$ is regular, we have $f=\delta_{k+1}(f) z^{k+1}+o\left(|z|^{k+1}\right)$, and so $f$ does not vanish on some deleted neighbourhood of 0 in $X$. As in Example 2.6 of (3), there exist $g, h \in D^{n}(X)$ such that $f=g h, g(0) \neq 0$, and $h(\zeta) \neq 0 \quad(\zeta \in X \backslash\{0\})$. Since $f(0)=0, h(0)=0$. The remaining assertions follow from the Leibnitz identities.

Note that assumption (A), together with the assumption that $X$ does not separate the plane, implies that a non-degenerate point derivation at 0 on $D^{n}(X)$ has order at most $2 n$ : compare (3), Example 2.6.

Finally, we consider the problem of showing that $z^{n} M_{n, n} \subset M_{n, n}^{2}$. The constructive proof given in the case of the unit interval cannot be adapted to the plane sets satisfying (A) that we are now considering. However, a modification of an argument originally due to P. C. Curtis does give the result. Set $X_{0}=X \backslash\{0\}$, and write $A_{n}$ for the set $\left\{f / z^{n}: f \in M_{n, n}\right\}$ of functions on $X_{0}$. Calculations given in (1), Theorem 2.1, show that $f \in A_{n}$ if and only if $f$ has $n$ continuous derivatives on $X_{0}$ and $\zeta^{k} f^{(k)}(\zeta) \rightarrow 0$ as $\zeta \rightarrow 0$ in $X_{0}(k=0, \ldots, n)$. Moreover, $A_{n}$ is a commutative Banach algebra with respect to the norm

$$
\|f\|=\sum_{k=0}^{n} \frac{1}{k !} \sup \left\{\left|\zeta^{k} f^{(k)}(\zeta)\right|: \zeta \in X_{0}\right\}
$$

The algebra $A_{n}$ cannot have an identity, but, if it has a bounded approximate identity, then we can conclude as in (1) and (3) that, given $f \in M_{n, n}$, there exist $g, h \in M_{n, n}$ such that $f / z^{n}=\left(g / z^{n}\right)\left(h / z^{n}\right)$, whence $z^{n} f=g h$. Thus, if $A_{n}$ has a bounded approximate identity, we obtain the inclusion $z^{n} M_{n, n} \subset M_{n, n}^{2}$, and so the following lemma provides the result that we require.

Lemma 3.2. Let $X$ be a compact plane set satisfying condition (A) which does not separate the plane. Then the algebra $A_{n}$ has a bounded approximate identity.

Proof. The point 0 lies on either one or two arcs, each continuously differentiable with respect to arc length. Thus, there is a continuous function $h:[0, \infty) \rightarrow C$ with $h(0)=0, h([0, \infty)) \cap X=\{0\},|h(t)| \rightarrow \infty$ as $t \rightarrow \infty$, and such that the argument of $h(t)$, measured continuously from 0 , is bounded-say $|\theta(t)| \leqq \frac{1}{3} N \pi$ for some $N \in N$, where $\theta(t)=\arg h(t)$. Write $H$ for the cut of the plane from 0 to $\infty$ defined by $h$.

We define $\zeta \mapsto \zeta^{1 / N}$ as an analytic function on $C \backslash H$ taking values in the sector $S=\left\{\zeta:|\arg \zeta| \leqq \frac{1}{3} \pi\right\}$, and we set $w(\zeta)=\zeta^{-1 / N}$ for $\zeta \in C \backslash H$. Note that we have $w^{\prime}(\zeta)=$ $-w(\zeta)^{N+1} / N$. For $m=1,2, \ldots$, define

$$
f_{m}(w)=\frac{m}{w}\left(1-e^{-w / m}\right) \quad(w \neq 0)
$$

and define $F_{m}$ by

$$
F_{m}(\zeta)=f_{m}(w(\zeta)) \quad\left(\zeta \in X_{0}\right)
$$


It is straightforward to show by induction on $k$ that

$$
F_{m}^{(k)}(\zeta)=C_{k} w^{k N}\left[f_{m}(w)-p_{k}\left(\frac{w}{m}\right) e^{-w / m}\right] \quad\left(k=1,2, \ldots, \zeta \in X_{0}\right),
$$

where $C_{k}$ is a constant and $p_{k}$ is a polynomial of degree $k-1$ with $p_{k}(0)=1$.

Now define functions $u_{k}$ by

$$
u_{k}(t)=\frac{1}{t}\left(1-e^{-t}\right)-p_{k}(t) e^{-t} \quad(t \in C \backslash\{0\}) .
$$

Then, from (3.1) it follows that

$$
\zeta^{k} F_{m}^{(k)}(\zeta)=C_{k} u_{k}\left(\frac{w}{m}\right) \quad\left(k=1,2, \ldots, \zeta \in X_{0}\right)
$$

In fact, (3.2) also holds for $k=0$ if we set $p_{0}=0$ and $C_{0}=1$. Note that $u_{k}(t) \rightarrow 0$ as $t \rightarrow \infty$ in $S$ and that $u_{k}(t) \rightarrow 0$ as $t \rightarrow 0$ in $S(k=1,2, \ldots)$, while $u_{0}(t) \rightarrow 1$ as $t \rightarrow 0$ in $S$.

We claim that $\left(F_{m}: m=1,2, \ldots\right)$ is a bounded approximate identity for the algebra $A_{n}$. First observe that $w / m \rightarrow \infty$ in $S$ as $\zeta \rightarrow 0$ in $X_{0}$, and so (3.2) implies that $\zeta^{k} F_{m}^{(k)}(\zeta) \rightarrow 0$ as $\zeta \rightarrow 0$ for each $k$ and $m$. Thus $\left(F_{m}\right) \subset A_{n}$. Also, using (3.2) again, the sequence $\left(F_{m}\right)$ is bounded in $A_{n}$. Now take $G \in A_{n}$ and $k \in\{0,1, \ldots, n\}$. Then, for $\zeta \in X_{0}$,

$$
\zeta^{k}\left(G F_{m}-G\right)^{(k)}(\zeta)=\zeta^{k} G^{(k)}(\zeta)\left(F_{m}(\zeta)-1\right)+\sum_{r=1}^{k} \zeta^{r} F_{m}^{(r)}(\zeta) \zeta^{k-r} G^{(k-r)}(\zeta)
$$

the sum in the above expression being taken to be 0 when $k=0$. Given $\epsilon>0$, we can choose $\delta>0$ so that $\left|\zeta^{k} G^{(k)}(\zeta)\right|<\epsilon$ for $k=0, \ldots, n$ provided that $\zeta \in X_{0}$ and $|\zeta|<\delta$. Also if $|\zeta| \geqq \delta$ and $\zeta \in X_{0}$, then $|w(\zeta)| \leqq \delta^{-1 / N}$, and so for all sufficiently large $m$, $\left|F_{m}(\zeta)-1\right|=\left|u_{0}(w / m)-u_{0}(0)\right|<\epsilon$ and, for $k=1, \ldots, n$,

$$
\begin{aligned}
\left|\zeta^{k} F_{m}^{(k)}(\zeta)\right|= & \left|C_{k}\left(u_{k}\left(\frac{w}{m}\right)-u_{k}(0)\right)\right| \\
& <\epsilon .
\end{aligned}
$$

Thus, using (3.3) in the two cases that $|\zeta|<\delta$ and that $|\zeta| \geqq \delta$, we see that $\left\|G F_{m}-G\right\| \rightarrow$ 0 as $m \rightarrow \infty$, as required.

This completes the proof.

The above lemma concludes the discussion of the four groups of technicalities, and the proof of Theorem 3.3 of (3) now gives the following result.

Theorem 3.3. Let $X$ be a compact plane set which does not separate the plane. Suppose that $X$ satisfies condition (A) and that 0 is a boundary point of $X$. If $d_{1}, \ldots, d_{p}$ is $a$ nondegenerate point derivation at 0 on $D^{n}(X)$, and if $p \geqq 2 q$, then the point derivation $d_{1}, \ldots, d_{\mathrm{q}}$ is continuous.

In fact, each of the other results of (3) concerning point derivations on $C^{(n)}([0,1])$ has a formally equivalent counterpart for algebras $D^{n}(X)$, where $X$ satisfies the 
conditions of the above theorem. It would perhaps be interesting to find a more complicated regular set $X$ for which the above results were not true.

\section{REFERENCES}

(1) W. G. BADE and P. C. Curtis, Jr., The structure of module derivations of Banach algebras of differentiable functions, J. Functional Analysis 28 (1978), 226-247.

(2) H. G. Dales and A. M. Davie, Quasianalytic Banach function algebras, J. Functional Analysis 13 (1973), 28-50.

(3) H. G. Dales and J. P. MCClure, Higher point derivations on commutative Banach algebras, I, J. Functional Analysis 26 (1977), 166-189.

(4) H. G. Dales and J. P. McClure, Higher point derivations on commutative Banach algebras, II, J. London Math. Soc. (2) 16 (1977), 313-325.

(5) J. Detraz, Sous-algèbres de codimension 1 et dérivations dans les algèbres de Banach commutatives, Studia Math. 30 (1968), 79-82.

(6) A. NAGEL, Cohomology of sheaves of holomorphic functions satisfying boundary conditions on product domains, Trans. Amer. Math. Soc. 172 (1972), 133-141.

(7) A. G. O'FArrell, Point derivations on an algebra of Lipschitz functions, Proc. Royal Irish Academy, Section A 80 (1979), 23-39.

(8) D. R. Sherbert, The structure of ideals and point derivations in Banach algebras of Lipschitz functions, Trans. Amer. Math. Soc. 111 (1964), 240-272.

SCHOOL OF MATHEMATiCS

UNIVERSITY OF LEEDS

LEEDS, LS2 9JT

ENGLAND

Department of Mathematics and Astronomy

UNIVERSITY OF MANITOBa

WINNIPEG, R3T 2N2

Canada 